\title{
Advanced Methods for Natural Product Drug Discovery in the Field of Nutraceuticals
}

\author{
Jean-Luc Wolfender*, Philippe J. Eugster, Nadine Bohni, and Muriel Cuendet
}

\begin{abstract}
Advances in analytical methods and bioassay development have helped to push forward the research in natural products. In plant extracts and nutraceuticals, bioactive compounds are part of a complex mixture. The development of high-resolution methods related to HPLC for both chemical and biological profiling has significantly increased the efficiency of classical bioactivity-guided fractionation procedures. Furthermore, the level of sensitivity obtained by these methods give the possibility to work with few micrograms of compound. This represents a key advantage for rapid localisation of the biological activity and subsequent identification of the compounds of interest. The same methods are also used to study the extracts from a metabolomic view point. The possibility to study them as a whole can highlight synergetic effects, which are likely to occur in plant extracts and nutraceuticals. In this paper, the main trends are summarised and the developments made in our laboratory on profiling crude extracts with UHPLC-TOF-MS, natural product identification at the microgram level using microflow NMR and integration of these methods with biological evaluation are highlighted.
\end{abstract}

Keywords: Biological activity · Metabolomics · Microflow NMR · Nutraceuticals · UHPLC-TOF-MS

\section{Introduction}

Medicinal plants, vegetables and food products are rich sources of natural compounds that may play a role in health. It can be by maintaining health, preventing or curing diseases. In medicinal plants, these compounds are considered as bioactive natural products and may ultimately be developed as drugs. In food, they would be defined as phytonutrients without having therapeutic claims but with significant health benefits that can be used in disease prevention. Phytonutrients are often nonessential food components and are consumed in large amounts by individuals in their everyday lives. ${ }^{[1]}$ Over the past 15 years, research on these positive effects has developed considerably to provide

${ }^{\star}$ Correspondence: Prof. J.-L. Wolfender Phytochimie et Produits Naturels Bioactifs Ecole de Pharmacie Genève-Lausanne Section des Sciences Pharmaceutiques Université de Genève

Quai Ansermet 30, 1211 Genève 4

Tel.: +412237933 85

Fax: +41223793399

E-mail: jean-luc.wolfender@unige.ch evidence to support a role in the prevention of various health problems, such as cardiovascular diseases and cancers. The structural complexity of phytonutrients is extremely vast and goes from simple phenols and phenolic acids to complex high-molecular-weight compounds, such as tannins and proanthocyanidins. They all have specific physicochemical properties. Clinical evidences on disease-risk reduction have been shown for a few food products. They are mainly due to their content in polyphenols as this class of chemicals is by far the most documented.

Recently, the term nutraceuticals, a term combining the words 'nutrition' and 'pharmaceutical', has emerged. It is defined as food or food components that provide health and medical benefits, including the prevention and treatment of diseases. Nutraceuticals can be found in a wide range of foods, from conventional to fortified or enriched foods, and also as dietary supplements. A dietary supplement is a product containing nutrients derived from food products that are concentrated in liquid or capsule form. The 'dietary ingredients' in these products may include: vitamins, minerals, herbs or other botanicals, amino acids, and substances increasing the total dietary intake, such as enzymes and organ tissues. However, despite the growing interest in herbal products, there is still no unified legislation. Indeed, phytonutrients from herbs and botanicals can be sold under various forms which would result in different regulations. On one hand, they can be consumed as capsules, powders, or pills, and in that case, they would be regulated as dietary supplements. On the other hand, they can be ingested as additives in conventional foods (e.g. teas, juices, chips) and would be considered as such. ${ }^{[2]}$ Since the legislation regarding the use of phytopharmaceuticals and traditional ingredients varies considerably from country to country, issues regarding their quality control and standardisation requirements remain open. Furthermore, the differences between phytopharmaceuticals and nutraceuticals are not always well defined. A striking example is that preparations containing Hypericum perforatum (Saint John's wort) are available as natural antidepressants only in pharmacies in Switzerland because of severe problems related to drug interactions. Similar extracts have been added to chocolate bars in the United States and are considered as dietary supplements which do not have to be approved by the U.S. Food and Drug Administration (FDA) before marketing.

\subsection{Relationship between Phytotherapy and Nutrition}

In modern approaches to nutrition and nutraceuticals, the search for bioactive compounds and the explanation of the mode of action of the phytonutrients within complex food products, is very similar to approaches used in pharmacognosy for studying medicinal plants. There are even cases when plants or enriched extracts used in phytotherapy are recognised as food supplements or nutraceuticals. They are often called functional foods and are 
designed to allow consumers to eat enriched foods close to their natural state, rather than by taking dietary supplements manufactured in liquid or capsule form. In the case of broccoli, super broccoli and BroccoSprouts ${ }^{\circledR}$ have been developed these past few years. They contain respectively 3.5 and 20 times more sulforaphane than usually produced by the vegetable.

Still several questions may be raised. Do certain kinds of food contain pharmacologically active substances in concentrations that are high enough to have drug-like effects when consumed? Are biologically active compounds in food indicative of therapeutic value? Is traditional drug development suitable for testing the merits of food? Is it ethical to test food as a drug on patients? Will dietary disease management remain a pipedream? ${ }^{[3]}$

\subsection{Cancer Chemoprevention Using Phytonutrients}

Dietary consumption of foods and herbal medicines is a convenient method of administrating potentially beneficial phytochemicals in a cost-effective manner. The benefits are even bigger for the prevention of chronic diseases, such as cancer, which implies long-term non-toxic treatment. Cancer is considered the end stage of a chronic disease process characterised by abnormal cell and tissue differentiation. This process of carcinogenesis eventually leads to the final outcome of invasive and metastatic cancer. Recent advances in defining cellular and molecular levels of carcinogenesis, along with a growing body of experimental epidemiological, and clinical trial data, have led to the development of cancer chemoprevention, a relatively new strategy in preventing cancer. ${ }^{[4]}$

Beside the control of causative exogenous factors, such as cigarette smoking, dietary factors, and specific microorganisms, cancer chemoprevention can play an integral role in the overall strategy geared toward reducing the incidence of cancer. ${ }^{[4]}$ Rational and successful implementation of chemopreventive strategies relies intrinsically on tests for efficacy and mechanistic assays, as well as availability of promising chemopreventive agents, reliable intermediate biomarkers, and appropriate clinical cohorts to discover safe and effective drugs for primary and secondary prevention of human cancers. Various foods have already shown promising results in this field.

\subsubsection{Cruciferous Vegetables}

Epidemiological evidence relating cancer risk reduction to the consumption of cruciferous vegetables such as broccoli, cauliflower, cabbage, kale, bok choy, Brussels sprouts, radish, or various mustards, has been summarised in several comprehensive reports. Highly significant cancer

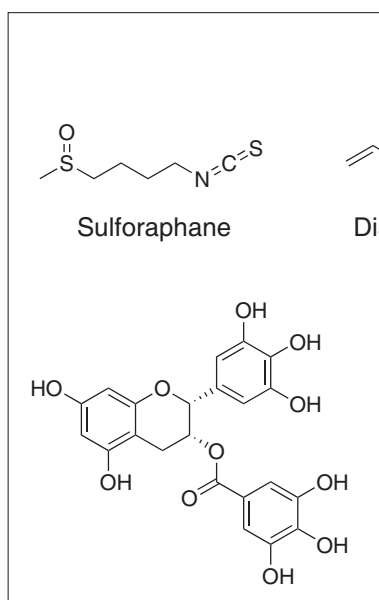

(-)-Epigallocatechin-3-gallate

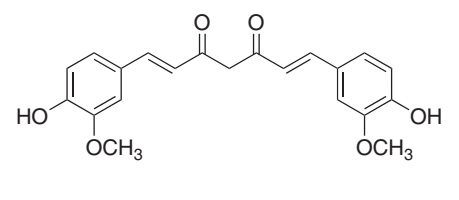

Curcumin

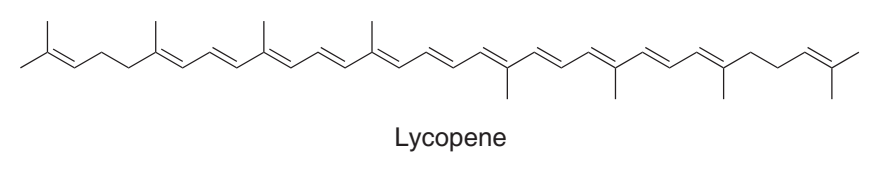

Fig. 1. Chemical structures of cancer chemopreventive agents isolated from food.

risk reduction with increasing crucifer intake was observed in cohorts that developed prostate, breast, bladder, and lung cancer, and non-Hodgkin's lymphoma. Some compounds are remarkably potent, such as sulforaphane (Fig. 1) and phenethyl isothiocyanate, components of broccoli and watercress, respectively. ${ }^{[5]}$

\subsubsection{Green Tea}

On a worldwide basis, the most popular chemopreventive drink is green tea. Green tea is the water extract of the dry leaves of Camellia sinensis. (-)-Epigallocatechin-3gallate (EGCG, Fig. 1), the most abundant catechin in green tea, is credited with the majority of health benefits associated with green tea consumption. ${ }^{6]}$ The organ sites where tea or tea constituents are found to be effective include the skin, lung, oral cavity, oesophagus, stomach, small intestine, colon, liver, prostate, and bladder. Despite large amounts of data, it is still not clear whether green tea has a real clinical efficacy. The possible complications of translating results obtained in cell culture studies to animals and humans may come from possible artefacts due to the auto-oxidation of EGCG. ${ }^{[7]}$ Also, activities observed in cell culture with high concentrations of EGCG may not be relevant because of the limited systemic bioavailability of EGCG.

\subsubsection{Allium Vegetables}

The large genus, Allium, includes the onion, garlic, chive, leek and shallot. Collectively, preclinical investigations demonstrate consistently that cancer chemoprevention by garlic and related sulfur compounds (Fig. 1) is clearly evident and appears to be independent of the organ site or the carcinogen employed. ${ }^{[8]}$ Some organoselenium compounds, also found in $\mathrm{Al}$ - lium spp, are superior to the corresponding sulfur analogs in cancer prevention.

\subsubsection{Tomato}

Epidemiological studies have provided evidence that high consumption of tomatoes effectively lowers the risk of reactive oxygen species (ROS)-mediated diseases such as cardiovascular disease and cancer by improving the antioxidant capacity. Tomatoes are rich sources of lycopene (Fig. 1), an antioxidant carotenoid reported to be a more stable and potent singlet oxygen quenching agent compared to other carotenoids. In addition to its antioxidant properties, lycopene showed an array of biological effects including cardioprotective, anti-inflammatory, antimutagenic and anti-carcinogenic activities. The anticancer activity of lycopene has been demonstrated both in in vitro and in vivo tumour models. ${ }^{[9]}$

\subsubsection{Red Wine}

Resveratrol (3,4',5-trihydroxystilbene, Fig. 1) is a phytoalexin produced in large amounts in grapevine skin in response to infection by Bothrytis cinerea. This production of resveratrol blocks the proliferation of the pathogen, thereby acting as a natural antibiotic. Numerous studies have reported interesting properties of trans-resveratrol as a preventive agent against various pathologies such as vascular diseases, cancers, viral infection or neurodegenerative processes. Several epidemiological studies have revealed that resveratrol is probably one of the main microcomponents of wine responsible for its health benefits. Resveratrol acts on the process of carcinogenesis by affecting the three phases: tumour initiation, promotion and progression phases and suppresses the final steps of carcino- 
genesis, i.e. angiogenesis and metastasis. Moreover, concentrations of resveratrol in blood seem to be sufficient for antiinvasive activity. Interestingly, low doses of resveratrol can sensitise to low doses of cytotoxic drugs and provide a strategy to enhance the efficacy of anticancer therapy in various human cancers.

\subsubsection{Curcuma}

Curcumin (Fig. 1), a yellow colouring ingredient present in turmeric (Curcuma longa), has emerged as one of the most powerful chemopreventive and anticancer agents. This compound has been shown to exert anti-carcinogenic effects in a diverse array of animal and cell culture models. It can act as a chemopreventive agent in cancers of colon, stomach and skin by suppressing colonic aberrant crypt foci formation and DNA adduct formation. ${ }^{[10]}$ Also, curcumin has been shown to down regulate $\mathrm{NF}-\kappa \mathrm{B}$ thereby suppressing proliferation and inducing apoptosis, and to possess anti-angiogenic properties. Cell lines that are resistant to certain apoptotic inducers and radiation become susceptible to apoptosis when treated in conjunction with curcumin.

All of these examples suggest that phytonutrients can be effective in the prevention of cancer or other diseases. Consequently, their specific bioactivity or their activity within complex matrices needs to be assessed in the same way as it is done with medicinal plants during the process of drug discovery. Thus, we are concentrating on the latest methodologies for profiling activity and chemical content in plant matrices to identify bioactive compounds. On the other hand, we apply the same tools to evaluate the biological effects of the whole mixture by taking into consideration their chemical complexity using metabolomic approaches.

\section{Improved Methods for Profiling Complex Mixtures Containing Phytonutrients}

As mentioned above, nutraceuticals and phytopharmaceuticals are closely related and a good understanding of their mode of action in terms of health benefit is needed. Unlike the case of herbal drugs or phytopharmaceuticals, the profiling of all constituents in food products is not required for their registration as nutraceuticals. Legislation varies considerably from country to country. However, for the understanding of the health benefits of such ingredients, many bioactive food components need to be analysed for complete identification or quantification in plant, fruit or vegetable matrices. This is also important for safety and compliance, especially when herbal additives are used in food products. Furthermore, the biological activity of the main ingredients has to be studied to support health claims and control the absence of toxicity. Thus, state-ofthe-art analytical methods and bioassays need to be developed taking into account the complexity of the matrices to study.

\subsection{Profiling Phytonutrients in Nutraceuticals}

For the analysis and profiling of these natural products either in plant materials or in body fluids, various methods have been described. They include mainly chromatographic techniques (HPLC, UHPLC, GC, HPTLC) and spectroscopic methods (MS, NMR) used either alone (HPLC-UV, HPLC-ELSD, GC-FID) or in hyphenation (LC-MS, GC-MS, LC-NMR). These methods can be used for targeted analysis (quantification, quality control, standardisation) or for phytonutrient profiling in complex matrices.

Profiling of phytonutrients in vegetables or traditional ingredients has been performed mainly with chromatographic methods based on HPLC. ${ }^{[11]}$ Various simple detectors such as UV or ELSD (Evaporative Light Scattering Detection) have been used, but mass spectrometry (MS), thanks to its sensitivity and selectivity, is undoubtedly the best choice for the detection of nearly all natural products. ${ }^{[12]}$

In this respect, we have been involved in many studies for the profiling and dereplication of natural products in complex matrices. Our latest applications imply the development of Ultra High Pressure Liquid Chromatography (UHPLC) methods for a high-resolution chromatographic separation of plant extract constituents combined to Time-Of-Flight Mass Spectrometry (TOF-MS) for sensitive detection and accurate mass determination. The recent introduction of sub-2 $\mu \mathrm{m}$ packing columns and UHPLC systems operating at very high pressures up to 1000 bars, led to a remarkable decrease in analysis time, higher peak capacity and increased sensitivity and reproducibility compared to conventional HPLC. [13] This technology gives a comprehensive characterisation of nutraceuticals by providing a very detailed high-resolution profiling (Fig. 2B \& D). At the same time it is used for high-throughput LC-MS fingerprinting (Fig. 2C). ${ }^{[14]}$ Such a methodology has recently enabled the profiling of the chemical constituents of various plant or vegetable matrices with high efficiency, ${ }^{[15]}$ and is also applied for metabolomic studies in this field (see below).

By selecting adequately the column length in UHPLC, it is possible, from a theoretical point of view, to increase the throughput by a factor 9 compared to con- ventional HPLC. On the other hand, by keeping strictly identical column lengths in both HPLC and UHPLC, it is hypothetically possible to increase the plate count by a factor 3 between columns ( $5 \mu \mathrm{m}$ vs $1.7 \mu \mathrm{m}$ particles). However, it becomes difficult to work in optimal flow rate conditions because of the important backpressure generated by long columns packed with sub- $2 \mu \mathrm{m}$ particles. Some separations involving $150 \mathrm{~mm}$ or even longer UHPLC columns have been reported in the literature and show very elevated efficiency. ${ }^{[15]}$

An example of the level of details a profile obtained by UHPLC or high-throughput fingerprinting provides, is shown for Ginkgo biloba in Fig. 2.[15] This plant is a widely used medicinal herb found in phytopreparations to enhance memory and concentration. Preparations of this plant have also been recently registered for their positive effect on delaying pathological symptoms of Alzheimer's disease. Most of the clinical studies supporting these health claims have been performed on standardised extracts. However, similar extracts are sold as nutraceuticals or are being part of food ingredients, such as chocolate bars called 'Unforgettables Dark Chocolate Bar' without rigorous control of safety and quality. Methods for the profiling of the composition of phytopharmaceuticals but also nutraceuticals are therefore required.

As shown in Fig. 2, a very detailed picture of the composition of the extract can be obtained. At the same time, the TOF-MS detection provides the molecular mass for each of the constituents of the mixture with high accuracy ( $<5 \mathrm{ppm})$ (Fig. 2E). This is extremely useful to determine the molecular formula of the various phytonutrients and to provide, together with chemotaxonomic information, a good way to fully or partially identify the metabolites of interest. This recent powerful high resolution profiling method in both chromatographic and mass spectrometric dimensions (Fig. 2D) is thus very useful for the characterisation of a given nutraceutical and can be applied to many vegetable matrices.

\subsection{Profiling Bioactivity}

To study nutraceuticals, it is important to obtain a detailed picture of their composition as described before, but also to obtain information on the biological activity of the various phytonutrients. Classically in pharmacognosy, such an approach has been made by bioactivity-guided fractionation. ${ }^{[16]}$ The biological activity is assessed directly on a crude extract by simple assays and is then followed during a multiple step chromatographic fractionation procedure of this complex mixture until pure bioactive compounds are isolated and characterised. This strategy has enabled the 


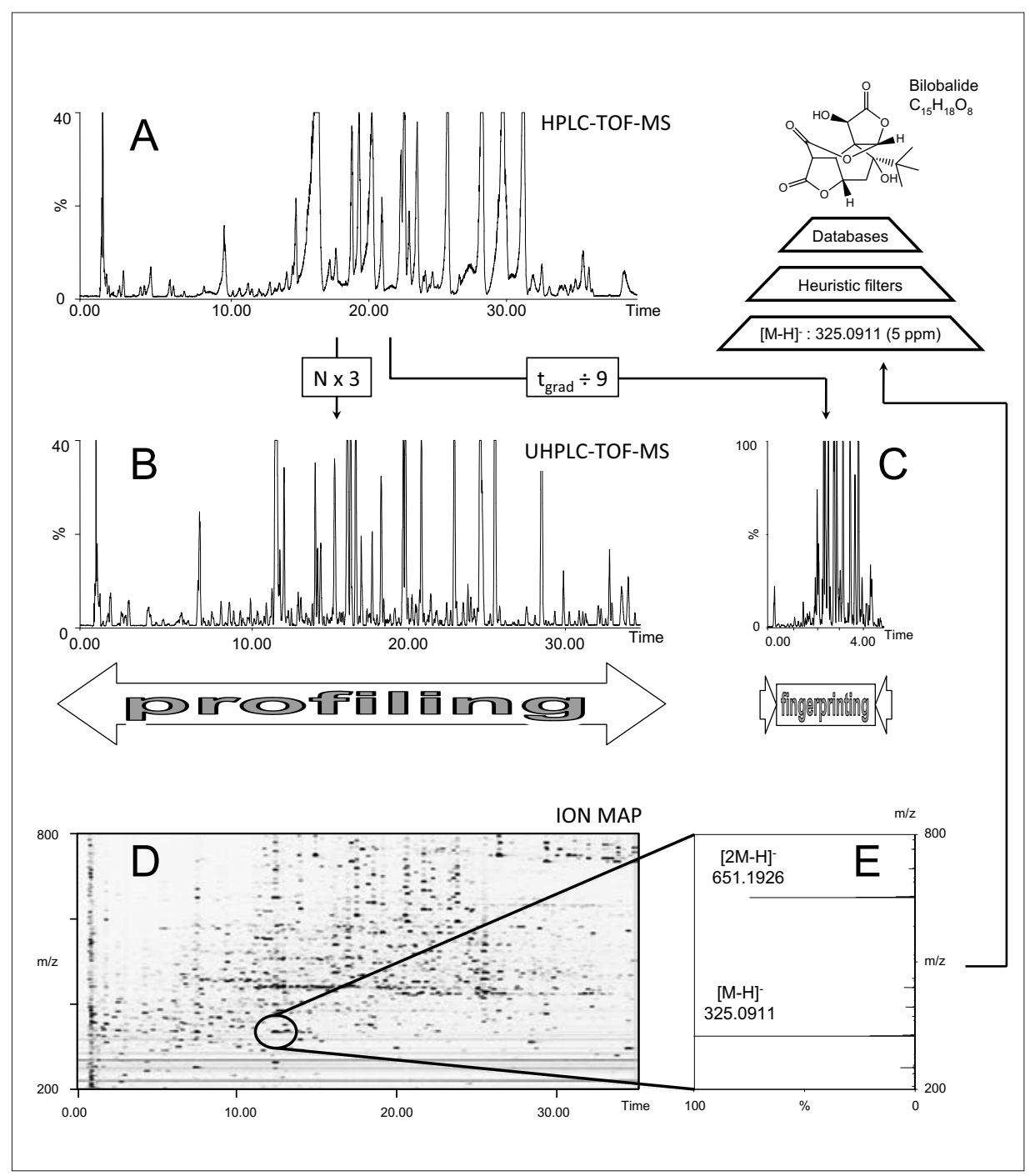

Fig. 2. Ginkgo biloba profiling: comparison of chromatograms (NI ESI-TOF-MS BPI traces) of a standardised Gingko biloba extract with transfer of method. A. Classical HPLC analysis carried out on a $5 \mu \mathrm{m}$ column $\left(150 \times 4.6 \mathrm{~mm}\right.$ i.d.) with gradient of $5-40 \% \mathrm{CH}_{3} \mathrm{CN}$ in $60 \mathrm{~min}$ at $1 \mathrm{ml} / \mathrm{min}$. B. HPLC method transferred to a $1.7 \mu \mathrm{m}$ UHPLC column $(150 \times 2.1 \mathrm{~mm}$ i.d., flow rate $0.35 \mathrm{ml} / \mathrm{min})$ with the same analysis time (increase of resolution, $\mathrm{ca} . \mathrm{N} \times 3$ ). C. Geometric transfer calculated by HPLC modelling software of the HPLC method on a short $1.7 \mu \mathrm{m}$ UHPLC column $(50 \times 2.1$ $\mathrm{mm}$ i.d., flow rate $0.6 \mathrm{ml} / \mathrm{min}$ ) (decrease of the gradient time: 9 -fold). D. UHPLC-TOF-MS 2D ion map (RT vs. $\mathrm{m} / \mathrm{z}$ ) of the separation on the $150 \mathrm{~mm}$ column. E. TOF-MS spectrum of bilobalide and molecular formula assignment procedure.

discovery of various bioactive compounds from medicinal plants, but also from food products, as described previously.

In this strategy, the path leading from the plant to its pure constituents is long. It involves work which might last anything from weeks to years. In order to rationalise and improve the efficiency of the approach, new strategies both in biological and chemical screening have to be developed in order to obtain valid chemical and bioactive information prior to starting the isolation work.

Improved methods for the localisation and characterisation of bioactivity remain thus a cornerstone for the study of phytonutrients, as well as for natural product-based drug discovery. Over the last decade, a wealth of new technologies
The assays that can be directly performed after TLC separation (bioautography) can be applied to many extracts or fractions at the same time. They are simple and have been widely used for a rapid localisation of bioactive compounds in extracts. Antioxidant and acetylcholinesterase inhibition activities can easily be evaluated by this method. ${ }^{[19,20]}$ However, TLC suffers from a lack of chromatographic resolution and the limited structural information that can be obtained on the compounds separated. The development of HPTLC which improves the resolution and the possibility to hyphenate TLC with MS detection will certainly push forward bioautography.

The assays that can be performed in connection with HPLC (on-line or at-line) are of special interest to us since they can nicely complement the detailed chemical profiles obtained by UHPLC-TOF-MS on crude extracts. Such approaches are often referred to as high-resolution screening. [21] An example is the development of HPLC on-line assays to test for antioxidant activity, which is of interest for nutraceuticals. This type of product often has health claims related to antioxidant properties since many chronic diseases (e.g. cancer, arteriosclerosis, Parkinson's disease, arthritis, etc.) are at least in part related to oxidative stress. On-line antioxidant activity can be measured by the post-column addition of a relatively stable coloured radical such as $\mathrm{DPPH}^{\circ}$ or $\mathrm{ABTS}^{-+}$. Radical scavengers are then detected as negative peaks because they reduce the model radical to its reduced, non-coloured form in a reaction coil. When combined with LC-MS and LC-NMR, reliable identification of active constituents becomes possible without the need of ever isolating them in a classical sense. ${ }^{[21]}$ Enzyme assays are, however, often not compatible with HPLC because of the organic modifier concentrations commonly used. Furthermore, the reaction time is often long and requires lengthy coils.

For performing rapid bioassays on HPLC peaks, at-line methods can be used. This involves the microfractionation of extracts in 96 well plates, plate drying to remove the solvent not compatible with the bioassays and dilution of the samples in a suitable solution or culture medium for performing the assay. Several examples of HPLC-based activity profiling in search of anti-inflammatory agents, monoamine oxidase inhibitors or GABA(A) modulators have demonstrated the efficiency of this approach. ${ }^{[22]}$ One of the bottlenecks of these approaches is the difficulty to quantify the potency of activity since the amount of natural products fractionated is often not known. Nevertheless this approach is very useful for a rapid localisa- 
tion of bioactive compounds in complex chromatograms, and for rationalising and improving the identification of active ingredients.

\subsection{Rapid Identification of Phytonutrients}

In complex food products, the characterisation of the phytonutrients and their metabolites also represents an important task. With HPLC or UHPLC mostly, MS or MS/MS spectra will be generated either with high or low resolution, depending on the instrumentation used. MS gives the possibility to generate nominal mass molecular ions or accurate mass measurements for the determination of empirical formulas. ${ }^{[23]}$ Furthermore, the use of tandem or hybrid MS instruments provides in-depth structural information through fragmentation of the molecular species by collision-induced dissociation (CID) reactions. ${ }^{[12]}$

For on-line identification purposes, the determination of the molecular weight is of great importance. This, however, requires the comparison of MS data obtained with different detection conditions in order to differentiate protonated or deprotonated molecules from adducts or fragments. ${ }^{24]}$ The use of high-resolution instruments, such as a TOF-MS or FTICR-MS system, enables the direct determination of the molecular formula of crude mixtures. The accuracy of MS measurement, usually below $5 \mathrm{ppm}$, and the ratio of intensities of the isotopic patterns, together with the use of rules for heuristic filtering of molecular formulae ${ }^{[25]}$ and chemotaxonomic information, allow an efficient mass-based putative metabolite identification and dereplication (Fig. 2E).

Complementary structural information can be generated by CID in LC-MS/ MS or $\mathrm{MS}^{\mathrm{n}}$ experiments. The generated CID spectra are, however, not comparable to those recorded by electron ionisation (EI), and this hampers a direct use of the standard EI-MS libraries for dereplication purposes. For analysis of fully unknown constituents, this approach usually cannot provide enough information to ascertain the structure, so that the combination with other on-line information is mandatory. For this, NMR used on-line (LC-NMR) or at-line (LC-SPE-NMR, microflow NMR) may contribute to the missing structural information. ${ }^{[24,26]}$ This method is, however, less sensitive and more time-consuming than MS, but is often mandatory to ascertain structure identification.

Considering all of these aspects, we have developed a strategy combining highresolution metabolite profiling of crude plant or vegetable extracts on high peak capacity UHPLC columns as shown in Fig. 2 with subsequent microfractionation of the extracts. The UHPLC conditions are transferred to the semi-preparative level using identical column stationary phase chemistry to ensure the same selectivity of separation (Fig. 3A \& B). LC-MS monitored microfractionation is done with milligrams of extracts or mixture (Fig. 3B \& C). Complete structural determination of the unknown compounds is then based on at-line microflow NMR (CapNMR ${ }^{\mathrm{TM}}$ ) experiments ${ }^{[27]}$ with detection at the microgram level (Fig. 3E). This approach provides high quality $1 \mathrm{D}$ and 2D NMR spectra on the main phytonutrients directly from a single separation of a crude extract, and minor constituents can be characterised after one further step of purification that can be optimised through software simulation and calculation. ${ }^{[28]}$

\subsection{Metabolite Identification and Bioactivity Assessment at the Microgram Level}

Crude extract profiling, chemical characterisation and bioactivity profiling can ideally be performed in an integrated manner. For this, we are developing a strategy that uses the microfractions described above for biological testing with various bioassays. Plants or nutraceutical extracts are microfractionated with amounts of material ranging from 10 to $100 \mathrm{mg}$. The microfractionation is directly performed in 96 deep well plates. The fractions containing typically 10 to 100 's of $\mu \mathrm{g}$ of pure or semi-pure natural products are aliquoted in other plates for bioassays while the main part of the microfractions is kept for further chemical characterisation. After mea-

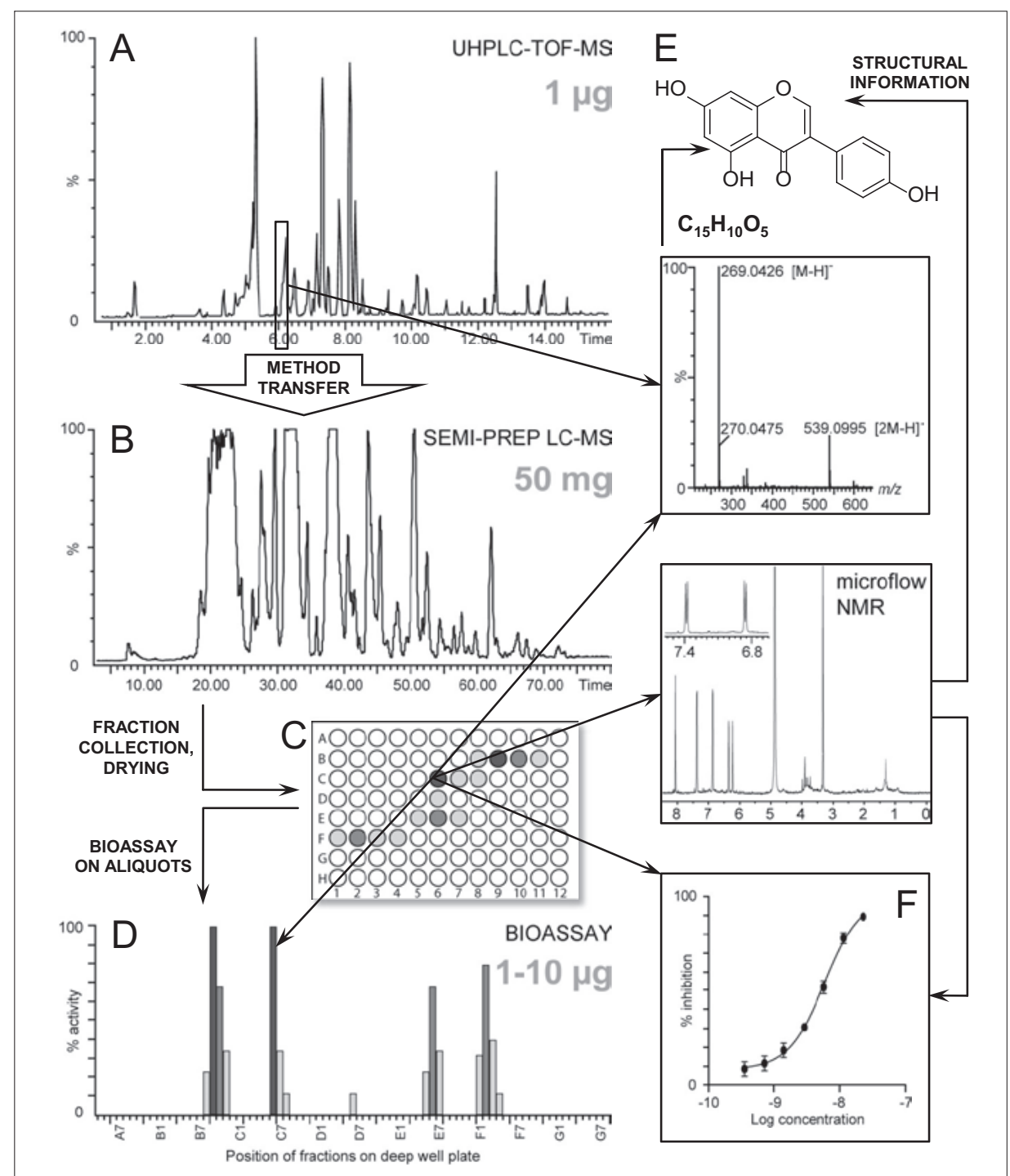

Fig. 3. Overview of the concept for metabolite identification and bioactivity assessment. A. Highresolution (HR) profiling of crude extracts by UHPLC-TOF-MS. B. Transfer of analytical conditions to the semi-preparative level to keep the same selectivity of separation (BPI traces are displayed). C. Microfractions are collected into deep well plates (one fraction/minute) and dried by vacuum centrifugation. D. Aliquots of all fractions are taken and analysed for their bioactivity. E. Microflow NMR analysis is performed on active microfractions to obtain structural and quantitative information. The molecular formula is extracted from the HR profiling $(A)$ as shown in Fig. 2F. The concentration of the compound present in the microfraction can be estimated using quantitative NMR. The activity is then evaluated by a dose-response curve. 
surement of the bioactivity, the wells of interest are analysed from the mother deep well plate by microflow NMR to perform metabolite identification together with molecular formula information extracted from the metabolite profiling. At the same time the amount of compound present in the fraction tested (typically a few micrograms) can be estimated by quantitative microflow NMR measurements (Fig. 3F). This enables the rapid localisation of bioactive compounds (Fig. 3C \& D), an estimation of the potency of the activity (Fig. $3 \mathrm{~F})$ and a complete or partial structure determination (Fig. 3E).

This concept has been validated in our search for phytotoxic ${ }^{[29]}$ and anti-inflammatory compounds using zebrafish assays in the 96 well format. ${ }^{[30]}$ Zebrafish have recently emerged as an attractive in vivo system for functional genomics drug discovery and can be used to assess toxicity. [31] Because of their small size, rapid development, optical transparency, and high genetic, physiologic, and pharmacologic similarities with humans, zebrafish embryos and larvae are an ideal model for studying bioactivity of natural product and phytonutrients already at the microgram scale. In our group, these activities are screened in collaboration with the University of Leuven (P. De Witte and A. Crawford). The microfractionation platform is presently optimised for searching for cancer chemopreventive agents from edible plants and vegetables by using a battery of short-term in vitro bioassays developed to monitor inhibition of tumorigenesis at the various stages. For initiation, induction of $\mathrm{NAD}(\mathrm{P}) \mathrm{H}$ :quinone reductase $(\mathrm{QR})$ activity, ${ }^{[32]}$ and antioxidant activity ${ }^{[33]}$ are assayed. Inhibition of inflammation factors such as NF- $\mathrm{KB}$ and nitric oxide, as well as the epigenetic activity are measured to assess the promotion stage. Finally, for progression, the evaluation of angiogenesis is performed.

\section{Reductionist versus Holistic Approaches to Study the Health Benefit of Phytonutrients}

As described above, the miniaturisation of the classical bioactivity-guided fractionation enables a significant increase of speed for tracking bioactivity and performing natural product identification. This type of investigation, however, is a reductionist approach and represents the current day paradigm of drug development: 'single target, single compound'. It involves thus mainly the testing of compounds at the molecular level in, for example, receptor binding assays. To study the mode of action or the health benefit of phytomedicine, nutraceuticals or food products, a more holistic approach using systems biology is probably much more suitable to prove efficacy, since all of these products represent complex mixtures of phytonutrients. ${ }^{[34]}$ These natural products can be prodrugs, have synergistic effects or other interactions with the rest of the complex matrices that can modify their pharmacodynamic properties. Synergy, prodrugs, and novel targets might be detected by a systems biology approach, whereas the reductionist approach will only recognise activity on already known targets, and will not detect synergism or prodrugs. In this respect metabolomics, a science that has emerged less than a decade ago, will be a major tool in recognising compounds connected to activity in complex food or phytotherapeutical products, and will also be very useful in evaluating the effects on the target organisms. It can be the patient in case of clinical trials with well-established traditional medicines ${ }^{[34]}$ or the consumer following a given diet or ingesting a food supplement for the study of nutraceuticals or food products.

In order to get a global view of all metabolites present in a plant, vegetable or body fluid after ingestion of these products, metabolomic studies can be performed either based on NMR or on MS fingerprinting. Today, the most advanced studies combine the advantages of MS and NMR spectroscopy. ${ }^{[35]} \mathrm{NMR}$ fingerprinting is used to analyse complex mixtures directly without the need of prior chromatographic separations. The method is indeed simple, has a high-throughput, does not require specific sample preparation, and provides detection of all protons in a quantitative manner. Standard protocols exist for the extraction and the analysis of various plant tissues, ${ }^{[36]}$ as well as for the study of biofluids. ${ }^{37]}$ The methods are extremely useful when a comparison of the major constituents of a plant extract is needed. The main drawback is that it lacks sensitivity and the identification of single compounds in extracts may be hindered by overlapping signals. Identification of biomarkers relies mainly on the comparison of NMR shifts of plant metabolites acquired in the same solvent conditions. De novo identification can be partly based on the acquisition of complementary 2D NMR experiments in the mixture.

MS provides a sensitive detection and the ability to identify metabolites based on MS/MS spectra when libraries are available. Because of its ability to analyse multiple analytes with a high sensitivity, MS is playing an increasingly important role in the progression of proteomics and metabolomics. Non-hyphenated MS methods enable the rapid and high-throughput screening of hundreds of samples, mainly for metabolite fingerprinting, but have limited quantification and metabolite identification capabilities. In hyphenation with HPLC or UHPLC, MS is extremely powerful in terms of detection, quantification and identification of a wide range of metabolites. It enables a bidimensional detection where each metabolite is resolved in both chromatographic (retention time) and mass spectrometric $(\mathrm{m} / \mathrm{z})$ dimensions, and high-throughput can be obtained with UHPLC-TOF-MS where extract fingerprinting can be generated typically in less than 10 minutes.

NMR or MS datasets are potential sources of information and in turn a source of knowledge. ${ }^{[38]}$ However, to make the leap from one to the other requires considerable data processing and statistical analysis. ${ }^{[39]}$ With the development of metabolomic methods for the analysis of complex biological systems, data on thousands of compounds are available in a single experiment and comparing samples has become a problem of high dimensionality. Extracting knowledge from these data, revealing patterns among samples and identifying critical or discriminatory variables is not a straightforward task. Because changes in metabolite levels may be drastic or subtle, important statistical processing is mandatory to determine the relevance of an observed change. Common chemometric tools, such as principal component analysis (PCA), used for projecting multivariate data to a low-dimensional plot, are generally proposed for display and exploratory analysis purposes. For further investigation, supervised approaches remain very attractive according to the strong impact of their use in human metabolomics. ${ }^{[39]}$

As an example of a MS based metabolomic approach on herbs and their effect on body fluid compositions, UHPLC-TOFMS has recently enabled the profiling of the chemical constituents of pu-erh tea, black tea, and green tea, as well as those of pu-erh tea products of different ages. Differences in tea processing resulted in differences in the chemical constituents and the colour of tea infusions. Human biological responses to pu-erh tea ingestion were also studied by UHPLC-TOFMS in conjunction with multivariate statistical techniques. Metabolic alterations during and after pu-erh tea ingestion were characterised by increased urinary excretion of 5-hydroxytryptophan, inositol, and 4-methoxyphenylacetic acid, along with a reduced excretion of 3-chlorotyrosine and creatinine. This study highlighted the potential of such a metabolomic approach to assess the effects of nutritional interventions containing phytonutrients on human metabolism. ${ }^{[40]}$

Similarly to this study, NMR-based metabolomic approaches have been reported for the classification of chamomile flowers 
from different geographic locations ${ }^{[41]}$ and for the detection of metabolites present in human urine samples. ${ }^{[42]}$

In our group, such approaches have been applied to the detection of low-abundance plant stress defence hormones using data mining applied to rapid UHPLC-TOFMS fingerprinting (Fig. 2C) and subsequent microfractionation of the biomarkers were highlighted and characterised by microflow NMR as described above. ${ }^{[43]}$ The same analytical strategy is currently being applied to study the effect of various phytopharmaceuticals with proven clinical efficacy for which classical reductionist bioactivity-guided fractionation approaches did not lead to the identification of the active ingredients. For such investigations, metabolomic data acquired on body fluids will be analysed from a holistic viewpoint after ingestion of the preparations and the bioactivity of metabolised phytonutrients will be measured.

\section{Conclusions}

As it is the case for bioactive natural products in medicinal plants, phytonutrients, as essential constituents of dietary supplements, play an increasingly important role in health issues. Thus the search for active ingredients in food products, nutraceuticals or medicinal plants is very similar and requires advanced methods for both chemical and bioactivity profiling. In this respect, recent evolution of LC-MS towards UHPLC-TOF-MS has given the possibility to investigate complex biological matrices with very high resolution and sensitivity, and to obtain a precise, but still incomplete, picture of the plant or vegetable metabolome. At the same time, development of NMR towards microNMR methods has boosted the sensitivity of this key technique for metabolite identification and allowed full phytochemical investigation at the microgram level. The integration of information rich bioassays at the same scale provides rapidly key information enabling the localisation and characterisation of bioactive constituents. The comprehension of the mode of action of each of these bioactive natural products is extremely complex and requires a thorough analysis of the constituents in their original matrices (fruits, vegetables, herbs or enriched extracts), as well as an assessment of their bioavailability. For this, metabolomics plays an increasingly important role. Recently, exciting research opportunities have appeared with the use of systems biology approaches to study the mode of action of medicinal plants or the health benefits of functional foods and food ingredients.

With the tremendous advancement in analytics, bioassays and data treatment over the last few years, it is safe to assume that research in natural products will continue to accelerate the pace at which significant discoveries are made in various health issues. However, this progress should also rely on a good understanding of the biological role phytonutrients have in vivo with all the possible biological targets they might interact with. These studies might ultimately lead to significant health benefits and represent a worthy challenge for natural product scientists within multidisciplinary research teams.

\section{Acknowledgements}

J. L. W. is grateful to the Swiss National Science Foundation for the financial support to the development of the miniaturised microfractionation and the metabolomics studies platform (Grant no. 205320-124667/1 and CRSII3_127187). J. L.W. is also grateful to the National Centre of Competence in Research (NCCR) Plant Survival, a research programme of the Swiss National Science Foundation for supporting the plant metabolomic platform.

Received: April 8, 2011

[1] J.-L. Wolfender, A. Violette, L. B. Fay in, 'Mass Spectrometry in Nutrition Research', Eds M. Kussmann, L. B. Fay, RSC, 2010, Vol. 9, pp 163 .

[2] S. S. Percival, E. Turner, in 'Handbook of Nutraceuticals and Functional Foods', Ed. R. E. C. Wildman, 2nd ed., CRC Press, Boca Raton, 2006, pp 269.

[3] M. Tulp, J. G. Bruhn, L. Bohlin, Drug Discov. Today 2006, 11, 1115.

[4] M. B. Sporn, N. Suh, Carcinogenesis 2000, 21, 525 .

[5] S. S. Hecht, Drug Metab. Rev. 2000, 32, 395.

[6] S. T. Saito, G. Gosmann, C. Pungartnik, M. Brendel, Recent. Pat. Food Nutr. Agric. 2009, 1, 203.

[7] C. S. Yang, J. D. Lambert, Z. Hou, J. Ju, G. Lu, X. Hao, Mol. Carcinog. 2006, 45, 431.

[8] L. M. Knowles, J. A. Milner, J. Nutr. 2001, 131, 1061S.

[9] T. Pannellini, M. Iezzi, M. Liberatore, F. Sabatini, S. Iacobelli, C. Rossi, S. Alberti, C. Di Ilio, P. Vitaglione, V. Fogliano, M. Piantelli, Cancer Prev. Res. 2010, 3, 1284.

[10] S. Singh, A. Khar, Anticancer Agents Med. Chem. 2006, 6, 259

[11] J.-L. Wolfender, Planta Med. 2009, 75, 719.

[12] M. Yang, J. H. Sun, Z. Q. Lu, G. T. Chen, S. H. Guan, X. Liu, B. H. Jiang, M. Ye, D. A. Guo, J. Chromatogr. A 2009, 1216, 2045.

[13] E. Grata, D. Guillarme, G. Glauser, J. Boccard, P. A. Carrupt, J. L. Veuthey, S. Rudaz, J.-L. Wolfender, J. Chromatogr. A 2009, 1216, 5660.

[14] J.-L. Wolfender, G. Glauser, J. Boccard, S Rudaz, Nat. Prod. Comm. 2009, 4, 1417.

[15] P. J. Eugster, D. Guillarme, S. Rudaz, J. L. Veuthey, P. A. Carrupt, J.-L. Wolfender, J AOAC Int. 2011, 94, 51.

[16] K. Hostettmann, A. Marston, Chimia 2007, 61, 322

[17] O. Potterat, M. Hamburger, Curr. Org. Chem. 2006, 10,899.

[18] H. L. Li, F. R. Song, J. P. Xing, R. Tsao, Z. Q. Liu, S. Y. Liu, J. Am. Soc. Mass Spectrom. 2009, 20, 1496.

[19] M. Cuendet, K. Hostettmann, O. Potterat, W. Dyatmiko, Helv. Chim. Acta 1997, 80, 1144.

[20] A. Marston, J. Kissling, K. Hostettmann, Phytochem. Anal. 2002, 13, 51.
[21] T. A. van Beek, K. K. R. Tetala, I. I. Koleva, A. Dapkevicius, V. Exarchou, S. M. F. Jeurissen, F W. Claassen, E. J. C. van der Klift, Phytochem. Rev. 2009, 8, 387.

[22] J. Zaugg, I. Baburin, B. Strommer, H. J. Kim, S. Hering, M. Hamburger, J. Nat. Prod. 2010, 73, 185.

[23] W. A. Korfmacher, Drug Discov. Today 2005 , 10, 1357.

[24] J.-L. Wolfender, G. Marti, E. F. Queiroz, Curr. Org. Chem. 2010, 14, 1808.

[25] T. Kind, O. Fiehn, Bmc Bioinformatics 2007, 8 , 115.

[26] J.-L. Wolfender, E. F. Queiroz, K. Hostettmann, in 'Bioactive Natural Products; Detection, Isolation and Structural determination', Eds. S. M. Colegate, R. J. Molyneux, CRC press, London, 2008, pp 143.

[27] A. Jansma, T. Chuan, R. W. Albrecht, D. L. Olson, T. L. Peck, B. H. Geierstanger, Anal. Chem. 2005, 77, 6509.

[28] G. Glauser, D. Guillarme, E. Grata, J. Boccard, A. Thiocone, P. A. Carrupt, J. L. Veuthey, S. Rudaz, J.-L. Wolfender, J. Chromatogr. A 2008 $1180,90$.

[29] G. Glauser, K. Gindro, J. Fringeli, J.-P. de Joffrey, S. Rudaz, J.-L. Wolfender, J. Agric. Food Chem. 2009, 57, 1127.

[30] A. Crawford, N. Bohni, J. Maes, A. Kamuhabwa, M. Moshi, C. Esguerra, P. de Witte, J.-L. Wolfender, Planta Med. 2010, 76, 1331.

[31] A. Crawford, C. Esguerra, P. de Witte, Planta Med. 2008, 74, 624.

[32] L. L. Song, J. W. Kosmeder, 2nd, S. K. Lee, C. Gerhauser, D. Lantvit, R. C. Moon, R. M Moriarty, J. M. Pezzuto, Cancer Res. 1999, 59, 578.

[33] H. S. Chung, L. C. Chang, S. K. Lee, L. A. Shamon, R. B. van Breemen, R. G. Mehta, N. R. Farnsworth, J. M. Pezzuto, A. D. Kinghorn, J. Agric. Food Chem. 1999, 47, 36.

[34] R. Verpoorte, Y. H. Choi, H. K. Kim, J. Ethnopharmacol. 2005, 100, 53.

[35] D. J. Crockford, A. D. Maher, K. R. Ahmadi, A. Barrett, R. S. Plumb, I. D. Wilson, J. K. Nicholson, Anal. Chem. 2008, 80, 6835 .

[36] H. K. Kim, Y. H. Choi, R. Verpoorte, Nat. Protocols 2010, 5, 536.

[37] J. C. Lindon, E. Holmes, J. K. Nicholson, FEBS J. 2007, 274, 1140.

[38] W. B. Dunn, D. I. Broadhurst, H. J. Atherton, R Goodacre, J. L. Griffin, Chem. Soc. Rev. 2011, $40,387$.

[39] J. Boccard, J. L. Veuthey, S. Rudaz, J. Sep. Sci. 2010, 33, 290.

[40] G. X. Xie, M. Ye, Y. G. Wang, Y. Ni, M. M. Su, H. Huang, M. F. Qiu, A. H. Zhao, X. J. Zheng, T. L. Chen, W. Jia, J. Agric. Food Chem. 2009, 57, 3046.

[41] Y. L. Wang, H. R. Tang, J. K. Nicholson, P. J. Hylands, J. Sampson, I. Whitcombe, C. G. Stewart, S. Caiger, I. Oru, E. Holmes, Planta Med. 2004, 70, 250.

[42] Y. L. Wang, H. R. Tang, J. K. Nicholson, P. J Hylands, J. Sampson, E. Holmes, J. Agric. Food Chem. 2005, 53, 191.

[43] E. Grata, J. Boccard, D. Guillarme, G. Glauser, P. A. Carrupt, E. Farmer, J.-L. Wolfender, S. Rudaz, J. Chromatogr. B 2008, 871, 261. 